\title{
Uterine Arteriovenous Malformations: A Rare Cause of Vaginal Hemorrhage after Hysterectomy
}

\author{
Jacques L*, Lund M and Baruah D
}

Department of Obstetrics and Gynaecology and Radiology, Medical College of Wisconsin, Milwaukee, Wisconsin, USA

${ }^{*}$ Corresponding author: Laura Jacques, Department of Obstetrics and Gynaecology, Medical College of Wisconsin, 9200 West Wisconsin Avenue, Milwaukee, WI 53226, USA, Tel: 414-805-6600; E-mail: ljacques@mcw.edu

Received date: 15 July, 2016; Accepted date: 26 August, 2016; Published date: 29 August, 2016

Citation: Jacques L, Lund M, Baruah D. Uterine Arteriovenous Malformations: A Rare Cause of Vaginal Hemorrhage after Hysterectomy. Gynecol Obstet Case Rep. 2016, 2:2

\section{Abstract}

Uterine arteriovenous malformations are rare causes of gynaecologic bleeding. The majority of cases are congenital, but at times can occur after obstetric or gynaecologic procedures, primarily uterine curettage or rarely hysterectomy. We present the case of a 27 -year-old female who experienced vaginal bleeding one month after a total laparoscopic hysterectomy as a result of a uterine arteriovenous malformation. Two attempts at selective embolization of the arteriovenous malformation were required prior to cessation of bleeding.

Keywords: Uterine arteriovenous malformation; Vaginal haemorrhage

\section{Introduction}

Uterine arteriovenous malformations (AVMs) are rare causes of gynaecologic bleeding. The incidence of uterine AVMs is unknown, but there have been less than 100 cases reported in the literature [1]. The etiology of uterine AVMs is either congenital or acquired. If congenital, these lesions are thought to occur as isolated, spontaneous failures of vascular development between the fourth and tenth week of embryogenesis.

The vascular malformation then grows along with and at the same rate as the individual but due to hormonal stimulation, such as puberty and pregnancy, may experience accelerated growth. These lesions may then become symptomatic due to enlargement of existing channels or recruitment of new channels resulting in increased flow rate or turbulence [2].

If acquired, AVMs tend to occur in reproductive age women after uterine curettage or rarely after hysterectomy. The largest systematic review identified 100 cases in the literature of AVMs diagnosed after uterine curettage. 96 of the 100 cases were premenopausal while the remaining 4 were postmenopausal; mean patient age was 301. The number of cases which have been reported after hysterectomy is far fewer.
A review of the literature identified 12 cases, the largest of which was a 1970 case series that included seven patients [3-5]. In all cases of AVM, either congenital or acquired, presentation is usually with large volume bleeding, anaemia, heart failure or a palpable, pulsatile mass on pelvic exam.

Diagnosis can be made with color Doppler pelvic ultrasound, angiography or MRI. Treatment historically consisted of surgical resection, but more recently has been successfully accomplished through interventional radiology uterine artery embolization. The authors report a case of a uterine AVM acquired soon after hysterectomy.

\section{Case Presentation}

A 27 year old null gravid female, anticoagulated on warfarin due to a mechanical tricuspid valve and underlying congenital heart disease, presented with sudden onset profus vaginal bleeding despite being on continuous oral contraceptive pills.

She was hospitalized on three separate occasions over a three month time period for management of menorrhagia, ultimately requiring a total of 25 units of blood transfusion. High dose intravenous estrogen was used successfully during her initial hospitalization and she was sent home on an oral contraceptive taper.

The bleeding resumed several weeks later, she presented to the hospital and a pelvic exam revealed a bluish mass at the left vaginal apex that was suspicious for an AVM. She was readmitted to the hospital. Due to the profuse nature of the bleeding, difficulty controlling it with hormonal methods, and question of a pulsatile mass in the vagina on examination, an MRI was done to evaluate for an AVM.

This imaging study showed blood products at varying stages expanding the endometrial cavity without any definite cause, and no AVM was noted (Figure 1).

She was then taken for a dilation and curettage and intraoperative levonorgestrel intrauterine device placement. The pathology showed benign endometrium with progestin effect. She was discharged home and did well for one week until she expelled the IUD and began bleeding again. She was managed initially as an outpatient by placing an etonogestrel implant. 


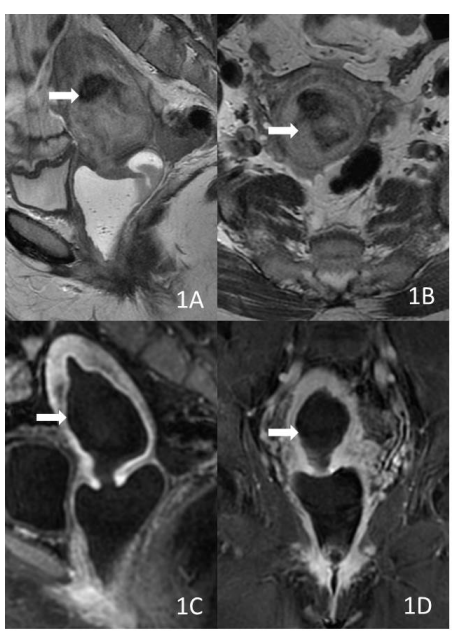

Figure $1 \mathrm{MR}$ image prior to hysterectomy. Images showing: Axial (1A) and Sagittal T2 weighted (1B) and sagittal (1C) and coronal (1D) post-contrast fat saturated T1 weighted MR images showing non-enhancing in homogeneous-intensity fluid in the uterus compatible with acute and sub-acute blood products (arrows). There is no enhancing mass lesion or evidence of vascular malformation.

One week later she was again admitted to the hospital with profuse vaginal bleeding, requiring transfusion. Therapy with depot leuprolide acetate was attempted without effect. Due to the failure of multiple hormonal treatments and the impact the bleeding was having on her cardiac function, the patient ultimately decided to proceed with a hysterectomy and bilateral salpingectomy. Her warfarin had been continued during this three month time period, keeping her INR in the therapeutic range of $2.5-3.5$, but was reversed prior to the hysterectomy.

She underwent an uncomplicated total laparoscopic hysterectomy with bilateral salpingectomy. Findings at the time of surgery were unremarkable; the uterus and pelvic anatomy appeared normal. Notably, there was no suspicion of an AVM at the time of surgery. The pathology showed no abnormalities of the uterus, cervix or fallopian tubes. She was restarted on a heparin drip and warfarin postoperatively until her INR was again in the therapeutic range.

She was discharged home and did well until 4 weeks postoperatively, when she presented to the emergency department with acute vaginal hemorrhage and syncope. Physical exam revealed an intact vaginal cuff and profuse bleeding, requiring use of wall suction for visualization. The bleeding was seen at the right side of the vaginal cuff.

The vagina was packed with gauze and she was sent for CT angiography of the pelvis. This revealed active arterial bleeding at the right vaginal apex and concern for an arteriovenous malformation (Figure 2). She was transfused two units of packed red blood cells, her anticoagulation was reversed using pro-thrombin complex concentrate (PCC), and she was taken to the interventional angiography suite where angiography demonstrated active extravasation from the right uterine artery with an artery-to-venous fistula (Figure 3).

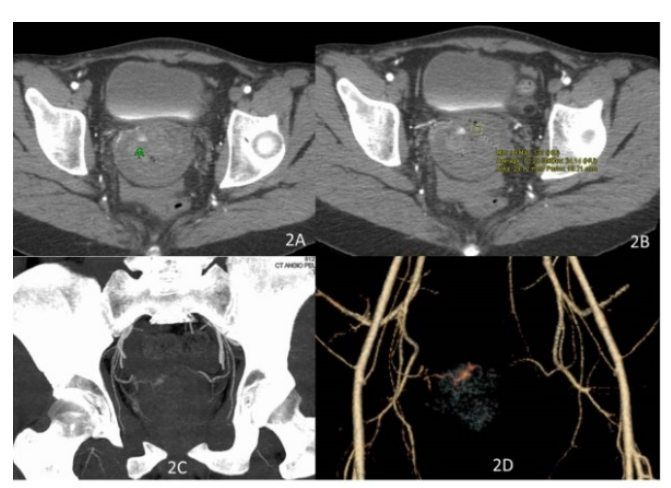

Figure 2 CT angiography done after hysterectomy prior to the first embolization by interventional radiology. Images showing high density (average Hounsfield Unit 73) acute blood/contrast in the pelvis (2B) with active arterial extravasation (2A). Active arterial extravasation is also seen nicely on maximum intensity projection (2C) and volume rendered (2D) images

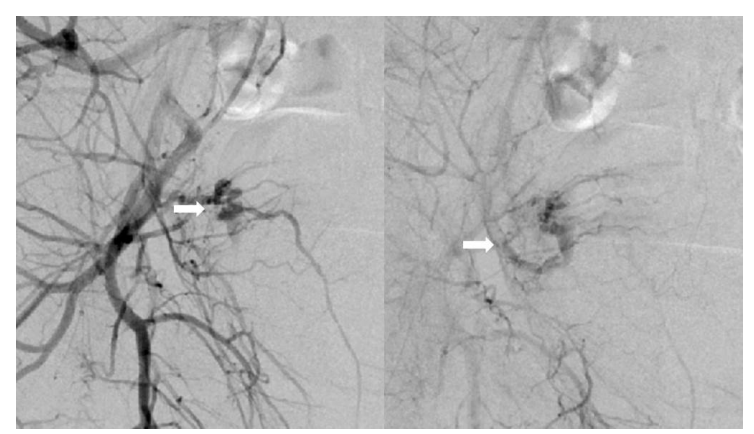

Figure 3 Angiography done at the time of the first uterine artery embolization by interventional radiology. Images showing: (3a). An arteriovenous fistula is noted involving the uterine artery in the catheter angiography (arrows) on right internal iliac artery injection corresponding to the CT angiography abnormality seen on Figure 2, (3b). Arrow showing the uterine artery was after embolization with coils and PVA (polyvinyl alcohol) particles

Two coils were placed proximal to the portion of the uterine vessel that had vaginal branches and resulted in successful occlusion and cessation of bleeding. She was restarted on her heparin drip and warfarin postoperatively, but on the day her INR reached the therapeutic range, she again started bleeding profusely from the vagina. PCC was used to reverse her anticoagulation. Right internal iliac arteriogram showing abnormal blush and arteriovenous fistula in same region as before, near the previously embolized right uterine artery (Figure 4). 


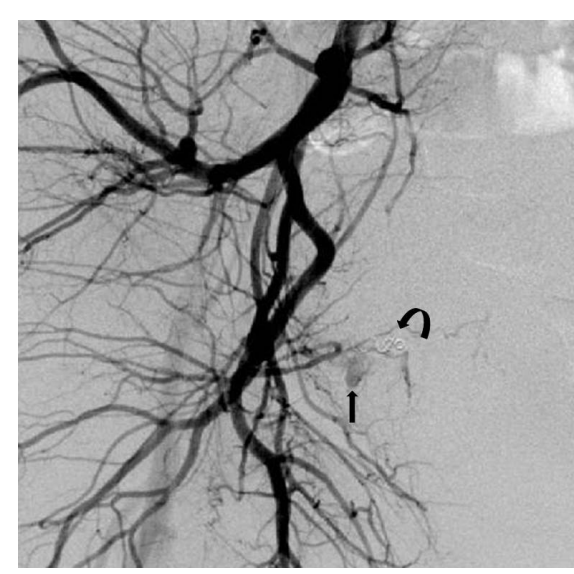

Figure 4 Right internal iliac artery catheter angiography done at the time of the second arterial embolization by interventional radiology. Images abnormal blush/ AV fistula (arrow) in the same location to previous embolization site (curved arrow showing the coil). Near stasis of the right uterine artery was noted which was further embolized completely with glue

The right uterine artery was completely embolized with glue. Catheterization of multiple pelvic branches did not reveal any contrast extravasation. She was restarted on warfarin and heparin drip 12 hours later, both of which were continued until her INR was in the therapeutic range. She was discharged home and had no further vaginal bleeding. Six-week postoperative examination revealed a well healed, intact vaginal cuff.

\section{Discussion}

Uterine arteriovenous malformations are extremely rare entities and those occurring after hysterectomy are even more so. Published cases after hysterectomy report symptoms from AVMs presenting anywhere from 3 months to 35 years postoperatively [3-6]. This case is unique in the short time interval from procedure to presentation. The proximity of the AVM presentation to hysterectomy created both etiologic and therapeutic uncertainties. Was the uncontrollable menorrhagia that lead to the hysterectomy actually due to a congenital AVM that went undiagnosed until now? Due to the proximity of surgery to the presentation of bleeding, would selective uterine artery embolization devascularized the vagina and lead to vaginal cuff dehiscence?.

Given the morbidity that laparotomy and ligation of the vessels would pose to this particular patient, it was determined that selective embolization was the optimal approach. Though concern still existed about the potential to decrease blood flow to the vaginal cuff and subsequent dehiscence, it was determined that the known risk of laparotomy for this anticoagulated patient was greater than this theoretical risk. Congenital AVMs tend to have an increased number of feeder vessels, and as such, have been described as more difficult to treat with embolization.
Acquired AVMs are more likely to have a limited number of contributing vessels and are therefore more amenable to endovascular intervention. Smith's review of the literature and case series (1994) reports that complete surgical resection appears to be permanently successful, though risks large volume blood loss at the time of surgery [3]. Selective embolization, by contrast, may have a higher failure rate due to incomplete obliteration and recruitment of collateral vessels leading to recurrence [2]. However, a more current review of 25 cases using selective arterial embolization to treat uterine AVMs states a success rate following one to two embolization procedures of $96 \%$ and a complication rate of $4 \%$.

As to the etiology of the AVM in this instance, there was a concern prior to hysterectomy for an AVM. Given the eventual diagnosis of arteriovenous fistula, this idea was again examined. The preoperative and postoperative images were reviewed after the fact and there was still no evidence of an AVM prior to the hysterectomy, nor was there any suggestion of this at the time of surgery or on pathologic examination. It is therefore concluded that this pathology occurred as a result of the hysterectomy. There has been a suggestion in the older gynaecologic surgical literature that transfixion ligation of the uterine artery pedicle is to be avoided due to the risk of creating an arteriovenous fistula [3-7]. In the vascular surgery literature, there are reports of AV fistula formation after endovenous treatment of varicose veins via radiofrequency ablation.

The proposed pathogeneses in this case are that heat transfer may cause microperforation and formation of $\mathrm{AV}$ fistula or that the inflammatory response from the procedure may lead to the formation of a small vessel network [8]. A bipolar vessel sealing device (Ligasure ${ }^{\mathrm{TM}}$ ) was used to obliterate the uterine vessels during this case. One possible etiology for the eventual AVM could be due to the use of this device. Sealing the vessels brings them into proximity and the heat and inflammation may lead to a micro perforation and subsequent fistulization. Even if the bipolar coagulator was involved in pathogenesis, many operations have employed this device, and a single case of AVM associated with its use does not suggest it should be abandoned.

This case serves as a reminder to include uterine arteriovenous malformation as a rare cause of posthysterectomy bleeding, and suggests that uterine artery embolization can be considered as a successful treatment strategy even in close proximity to the initial surgical event.

\section{References}

1. Peitsidis $\mathrm{P}$, Manolakos E, Tsekoura V, Kreienberg R, Schwentner $\mathrm{L}$ (2011) Uterine arteriovenous malformations induced after diagnostic curettage: a systematic review. Arch Gynecol Obstet 284: 1137-1151.

2. Rosen RJ, Riles TS (1994) Arterial venous malformations. In: Strandness DE, van Breda A (Eds). Vascular diseases. Surgical and interventional therapy 1121-1137. 
3. Smith Michael R (1995) Pulsatile pelvic masses: Options for evaluation and management of pelvic arteriovenous malformation. Am J Obstet Gynecol 1857-1863.

4. Sharma A, Vogelzang RL (2009) Uterine arteriovenous fistula as a long-term complication of hysterectomy. Obstet Gynecol 113: 489-491.

5. Fulmer GT, Mayberger HW, Sheehy TJ, Hayden CW (1970) Arteriovenous fistula of the uterine artery. A rare complication of hysterectomy. Angiology 21: 647-653.
6. Badawy SZ, Etman A, Singh M, Murphy K, Mayelli T (2001) Uterine artery embolization. The role in obstetrics and gynaecology. J clin imaging 25: 288-295.

7. Field CA, Welch JS, Johnson CE (1963) Posthysterectomy arteriovenous fistula involving uterine artery and vein. Am J Obstet Gynecol 87: 105-108.

8. Ahmad A, Sajjanshetty M, Mandal A, Hamilton H (2013) Early arteriovenous fistula after radiofrequency ablation of long saphenous vein. Phlebology 28: 438-440. 